\title{
Autoeficacia, Prácticas de Aprendizaje Autorregulado y Docencia para fomentar el Aprendizaje Autorregulado en un Curso de Ingeniería de Software
}

\author{
Carolina Zambrano \\ Escuela de Ingeniería, Universidad Católica del Norte, Larrondo 1281, Coquimbo, Chile. \\ (e-mail:carolinazambrano@gmail.com)
}

Recibido Oct. 5, 2015; Aceptado Dic. 7, 2015; Versión final Mar. 13, 2015, Publicado Jun. 2016

\begin{abstract}
Resumen
En este trabajo se presenta un estudio sobre la autoeficacia de los estudiantes y las practicas relacionadas con el proceso de aprendizaje autorregulado por parte de los estudiantes de un curso de ingeniería de software de la carrera de Ingeniería Civil en Computación e Informática de la Universidad Católica del Norte, Coquimbo, en Chile. El aprendizaje autorregulado es un proceso donde el estudiante autorregula su aprendizaje y toma conciencia de sus propios procesos cognitivos y socio-afectivos. Para realizar el estudio, se aplicaron dos instrumentos: la escala de autoeficacia general y un grupo focal (focus group) que permite describir las prácticas asociadas al aprendizaje autorregulado. Los resultados muestran que los estudiantes no planifican, ni monitorean su proceso de aprendizaje. Sin embargo, son capaces de evaluar y reflexionar sobre las causas del por qué no realizan una planificación y monitoreo. Se presenta una propuesta de promoción del aprendizaje autorregulado que permite instruir a los estudiantes en esta área.
\end{abstract}

Palabras clave: estrategias de aprendizaje; estrategias metacognitivas; aprendizaje autorregulado; autoeficacia general

\section{Self-efficacy, Practices of Self-Regulated Learning and Teaching Practices to promotion Self-Regulated Learning in a Course of Software Engineering}

\begin{abstract}
A study about self-efficacy and self-regulated learning practices of students of a course of Computing \& Informatics Engineering in a course of Software Engineering of the Universidad Católica del Norte, Coquimbo, in Chile. Self-regulated learning is a process in which students self-regulate their learning and become aware of their own cognitive and socio affective processes. For the study, two instruments were applied: the general self-efficacy scale and a focus group that would allow describing practices related to self-regulated learning. The results show that students do not plan nor monitor their learning process. However, they are able to assess and reflect on the reasons why they do not plan or monitor their learning process. A new proposal to promote self-regulated learning that allowed instruct students on this area is presented
\end{abstract}

Keywords: learning strategies; metacognitive strategies; self-regulated learning; general self-efficacy 


\section{INTRODUCCIÓN}

En la actualidad formar profesionales requiere de un constante perfeccionamiento y continua revisión tanto del mercado laboral como de los estándares nacionales e internacionales que pueden influir en los diseños curriculares y los diseños de modelos educativos de las organizaciones educacionales. También se debe considerar el estado actual del sistema de educación superior donde el ingreso ya no es restrictivo, sino que es masivo. Este último punto nos entrega un estudiante con características muy distintas, que es acogido por las instituciones educacionales y que debe ser retenido por los programas de nivelación y/o de apoyo inicial para cumplir con los estándares de acreditación respecto a la retención.

Por lo anterior el docente universitario actual (Monereo, 2009; Daura, 2011) es uno de los responsables de estimular en los estudiantes los conocimientos propios de la disciplina que enseña y además las competencias necesarias para el mundo laboral. Es necesario que el estudiante aprenda a aprender para que tenga la capacidad de ser autónomo para desenvolverse en el contexto académico y laboral. En este contexto el aprendizaje autorregulado cobra importancia. El aprendizaje autorregulado es un proceso donde el estudiante autorregula su aprendizaje y toma conciencia de sus propios procesos cognitivos y socio-afectivos (Zimmerman, 1989; Zimmerman, 1990; Zimmerman, 2001). Esta toma de conciencia es lo que se llama metacognición. El esfuerzo pedagógico en este caso está orientado hacia la formación de sujetos centrados en resolver aspectos concretos de su propio aprendizaje, y no sólo en resolver una tarea determinada, es decir, se debe orientar al estudiante a que se cuestione, revise, planifique, controle y evalué su propia acción de aprendizaje (Pintrich, 1990). Para Pintrich y Zimmerman la autorregulación implica tener conciencia del propio pensamiento, es el conocimiento acerca de cómo se aprende. Este modo más profundo de aprendizaje se desarrolla a través de observar en acción las propias conductas adoptadas para aprender.

Según Rosário el aprendizaje autorregulado se define como "un proceso activo en el cual los estudiantes establecen los objetivos que guían su aprendizaje intentando monitorizar, regular y controlar su cognición, motivación y comportamiento con la intención de alcanzarlos" (Rosario, 2004), y hace referencia a una concepción del aprendizaje centrada en los componentes cognitivos, motivacionales y conductuales que proporcionan al individuo la capacidad de ajustar sus acciones y metas para conseguir los resultados deseados teniendo en cuenta los cambios en las condiciones ambientales (Zeidner, 2000). Por lo anterior en el contexto de aprendizaje autorregulado se espera que el estudiante sea capaz de: i) Planificar: lo que implica establecer metas y actividades que posibiliten el cumplimiento de las tareas; ii) Monitoriar: incluye la comprensión de cómo se está realizando la tarea y la redirección de las estrategias que se utilizan, si fuese necesario; y iii) Evaluar: es la comprensión de la eficacia y la eficiencia con la que se desarrolla la actividad de aprendizaje. Permite valorar qué tanto el esfuerzo realizado corresponde con los resultados obtenidos.

Otro factor importante de considerar para comprender el aprendizaje autorregulado es la autoeficacia que influye en diversos aspectos de la autorregulación y el rendimiento. En este contexto la autoeficacia son las creencias que tiene una persona de poseer las capacidades para desempeñar acciones necesarias que le permitirán obtener los resultados deseados. La autoeficacia influye en como las personas piensan, sienten y actúan. Un alto sentido de eficacia facilita el procesamiento de información y el desempeño cognitivo en distintos contextos, incluyendo la toma de decisiones y el logro académico, así mismo los niveles de autoeficacia pueden aumentar o reducir la motivación de la persona. Bouffard (2005) estudió la interacción entre el rendimiento y el logro de aprendizajes en los estudiantes, con el fin de examinar cómo las creencias de eficacia intervienen sobre la autorregulación de la tarea cognitiva, corroborando que existe una asociación, tanto en hombres como mujeres.

Para evaluar el aprendizaje autorregulado existen instrumentos tales como SELF-A,CEPAA y MSLQ. SELFA es un cuestionario cuyo objetivo es evaluar percepciones de autoeficacia para el aprendizaje autorregulado sus autores son Zimmerman y Kitsantas (Zimmerman y Kitsantas, 2007). CEPAA es un cuestionario de estrategias para el aprendizaje autorregulado su propósito es evaluar un conjunto de estrategias para el aprendizaje autorregulado. MSLQ es un instrumento que permite obtener información sobre las variables cognitivas y motivacionales que influyen en el proceso de autorregulación (Pintrich, 1991). Tanto SELF-A, CEPAA y MSLQ no han sido validados para Chile. Por otro lado la Escala de Autoeficacia General de Baessler y Schwarzer mide las creencias de eficacia de los individuos (Baessler y Schwarzer, 1996). Para este estudio se decidió utilizar la Escala de Autoeficacia General debido a que es un instrumento que ha sido validado para Chile (Cid, 2010) y que además se ha mostrado que la autoeficacia influye en diversos aspectos de la autorregulación y el rendimiento (Bouffard, 2005).

Este trabajo se justifica dado que los estudiantes de Ingeniería Civil en Computación e Informática deben poseer una gran capacidad de autonomía debido a que las tecnologías de información cambian constantemente de enfoques; y el mercado laboral exige un nivel de aprendizaje autónomo elevado lo que obliga a los egresados a estar permanentemente actualizados en las nuevas herramientas y enfoques 
tecnológicos. Fue así que se detectó por parte de la docente una carencia del uso de estrategias de aprendizaje autorregulado lo que afecta el rendimiento de los estudiantes. Por lo anterior el objetivo de este trabajo es realizar un diagnóstico, y luego diseñar e implementar estrategias docentes que permitan la promoción del aprendizaje autorregulado en los estudiantes del curso de Ingeniería de Software de la carrera de Ingeniería Civil en Computación e Informática. Por otro lado, a futuro se podrá relacionar los resultados de esta investigación en curso con investigaciones de análisis de rendimiento (Zambrano, 2011; Zambrano, 2012).

En el contexto del diagnóstico se aplicaron dos instrumentos; la Escala de Autoeficacia General para detectar su nivel de tolerancia a situaciones estresantes y se realizó un grupo de discusión para detectar las prácticas de estudio relacionadas con el aprendizaje autorregulado que los estudiantes practicaban. Posteriormente se presenta una propuesta de promoción del aprendizaje autorregulado basada en la inclusión curricular ya que las actividades se desarrollan en la misma clase y se diseñan en la planificación didáctica del curso.

En relación con otros estudios sobre diagnósticos en el ámbito de aprendizaje autorregulado se puede mencionar el trabajo de Pool-Cibrian y Martínez- Guerrero donde se evalúa la relación entre la autoeficacia percibida, metas de aprendizaje y estrategias para el aprendizaje autorregulado (Pool-Cibrian y MartínezGuerrero, 2013) usando los instrumentos SELF-A y CEPAA a una muestra de 766 estudiantes universitarios de distintas carreras. Por otro lado Daura (2015) presenta un trabajo que esta focalizado en estudiantes de medicina donde se investigó las variables relacionadas entre el aprendizaje autorregulado y el rendimiento en dichos estudiantes. En Chile el trabajo de Díaz et. al. (2010) muestra un estudio de los procesos de autorregulación del aprendizaje en estudiantes universitarios de primer año. Por otro lado la actividad didáctica del docente puede ser ajustada con el objetivo de promover en los estudiantes el aprendizaje autorregulado como un proceso de planificación, monitoreo y evaluación permanente (Biggs, 2001; Cochran-Smith, 2003, Perez et al., 2010). En este contexto existen dos modalidades para la aplicación de programas y/o intervenciones para la promoción de los aprendizajes autorregulados estos son, programas extracurriculares y programas de inclusión curricular (Entwistle y Tait, 1992).

\section{METODOLOGÍA}

Según Namakforoosh (2011) el tipo de investigación que se llevó a cabo fue de tipo cualitativo y cuantitativo ya que se aplicaron dos instrumentos uno para cada caso. En el caso cuantitativo se enfocó a recopilar información acerca de creencias de eficacia de los estudiantes aplicándose el test de autoeficacia general. Para el caso cualitativo se realizó un grupo de discusión que permitió recabar información sobre las prácticas de estudio de los estudiantes asociados al proceso de aprendizaje autorregulado (planificar, monitorear, evaluar). La técnica de análisis cualitativa utilizada es el análisis de contenido.

Muestra: La muestra constó de 15 estudiantes, 13 hombres y 2 mujeres, los que en el momento de la evaluación cursaban la asignatura de Ingeniería de Software, perteneciente a la carrera de Ingeniería Civil en Computación e Informática. La edad promedio de los estudiantes fue de 22 años. Los estudiantes presentaban atraso en el avance de su carrera debido a reprobación de una o más asignaturas. Todos declararon estar motivados y a gusto con su carrera. Esta motivación y gusto por su carrera se refleja en que los 15 estudiantes presentaban un avance de 4 años académicos de carrera y ya habían aprobado los semestres y asignaturas que según las estadísticas de reprobación son asignaturas críticas (física III, cálculo III y programación I).

Escala de Autoeficacia General: La aplicación de este instrumento se realizó de forma física a través de papel y lápiz a 15 estudiantes. El instrumento Escala de Autoeficacia General (Baessler y Schwarzer, 1996) consta de 10 ítems y permite medir la percepción de una persona al afrontar una variedad de situaciones estresantes. El estudiante debe indicar si está de acuerdo con las afirmaciones, las cuales van desde "totalmente en desacuerdo" (0) hasta "totalmente de acuerdo" (3). La Tabla 1 muestra los Items de la Escala de Autoeficacia General.

Grupo de discusión: Se generó un guión que permitiera levantar información sobre las prácticas de estudio que estén relacionadas con el aprendizaje autorregulado. Se exploraron tres dimensiones (Zimmerman, 2000, 2002): i) Describir las prácticas de planificación de estudio por parte de los estudiantes; ii) Describir las prácticas de monitoreo de estudio por parte de los estudiantes: y iii) Describir las prácticas de evaluación de estudio por parte de los estudiantes. El grupo de discusión se aplicó a 13 estudiantes. Se realizó una semana después de la aplicación del instrumento Escala de Autoeficacia. Duró 25 minutos aproximadamente y fue dirigido por la psicóloga Katherine Campusano de CIMET UCN sede Coquimbo, en el contexto del acompañamiento pedagógico que CIMET otorgó a esta investigación. 
Tabla 1. Items Escala de Autoeficacia

\begin{tabular}{|l|l|}
\hline$N^{\circ}$ Ítem & Afirmación del ítem \\
\hline 1 & Puedo encontrar la manera de obtener lo que quiero aunque alguien se oponga. \\
\hline 2 & Puedo resolver problemas difíciles si me esfuerzo lo suficiente. \\
\hline 3 & Me es fácil persistir en lo que me he propuesto hasta llegar a alcanzar mis metas. \\
\hline 4 & Tengo confianza en que podría manejar eficazmente acontecimientos inesperados. \\
\hline 5 & Gracias a mis cualidades y recursos puedo superar situaciones imprevistas. \\
\hline 6 & $\begin{array}{l}\text { Cuando me encuentro en dificultades puedo permanecer tranquilo/a porque cuento con las habilidades } \\
\text { necesarias para manejar situaciones difíciles. }\end{array}$ \\
\hline 7 & Venga lo que venga, por lo general soy capaz de manejarlo. \\
\hline 8 & Puedo resolver la mayoría de los problemas si me esfuerzo lo necesario. \\
\hline 9 & Si me encuentro en una situación difícil, generalmente se me ocurre qué debo hacer \\
\hline 10 & Al tener que hacer frente a un problema, generalmente se me ocurren varias alternativas de cómo resolverlo. \\
\hline
\end{tabular}

\section{RESULTADOS}

\section{Escala de autoeficacia general}

En este trabajo la autoeficacia se ha conceptualizado de manera general. Se refiere a un sentido amplio y estable de la competencia personal sobre cuán efectiva puede ser la persona al afrontar una variedad de situaciones estresantes. Es importante recordar que se utilizó la Escala de Autoeficacia General debido a que se ha mostrado que la autoeficacia influye en diversos aspectos de la autorregulación y el rendimiento académico (Bouffard, 2005). A continuación se presentan los resultados obtenidos.

Como se observa en la Figura 1 el puntaje obtenido en la Escala de Autoeficacia General, está por sobre la media teórica $(\mathrm{MT})$ con $\mathrm{MT}=1,5$. Así también cada uno de los ítem analizados están por sobre la MT, sin embargo se puede observar que el ítem 4 "Tengo confianza en que podría manejar eficazmente acontecimientos inesperados", obtiene una media experimental $(M)$ igual a $M=1,86$ con $D T=0,66$. Es decir, la media experimental esta sólo tres puntos sobre la MT lo que indica que los estudiantes dudan de su eficacia en situaciones imprevistas. Por otro lado, el ítem 8 "puedo resolver la mayoría de los problemas si me esfuerzo lo necesario", obtiene una media experimental $M=2,64$ con $D T=0,50$, es el ítem que puntúa más alto en comparación con los otros ítem, lo que indica que los estudiantes atribuyen importancia al logro de sus objetivos al esfuerzo que pongan en lograrlo. Los detalles por cada ítem se observan en la Figura 1.

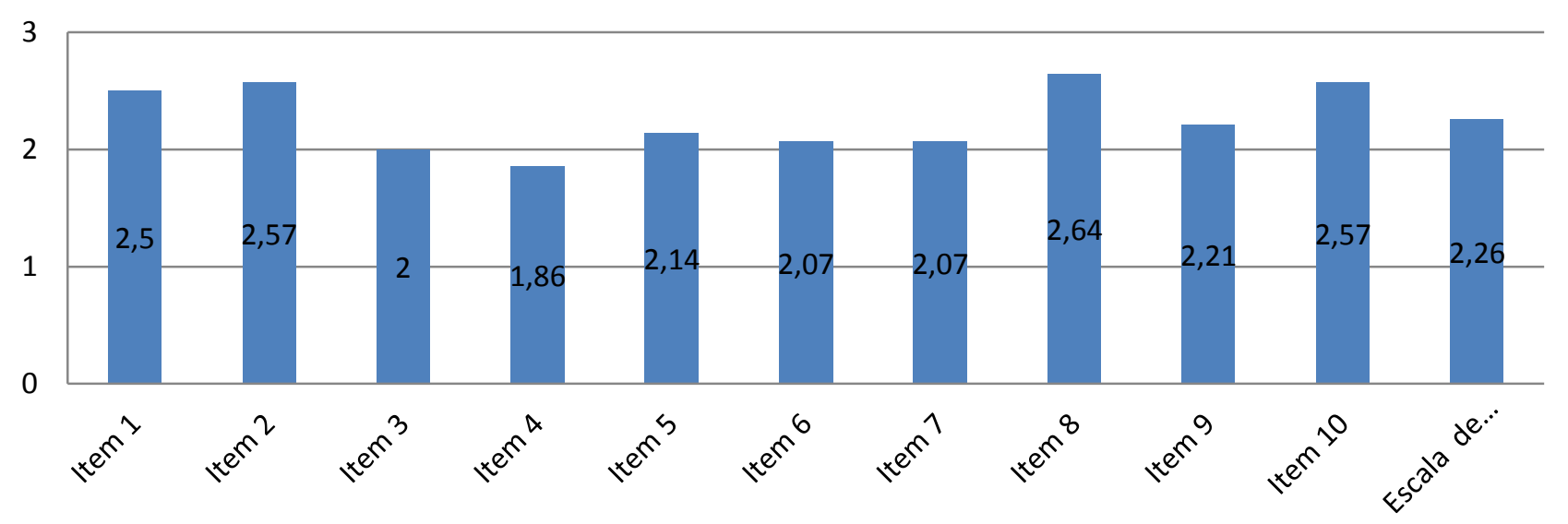

Fig. 1: Medias por Item de Escala de Autoeficacia General

\section{Aplicación grupo de discusión}

En el grupo de discusión las preguntas que se realizaron perseguían pesquisar: I) Las prácticas de planificación de estudio; II) Las prácticas de monitoreo de estudio; y III) Las prácticas de evaluación de estudio. Los resultados se agruparon en 17 categorías que se describen a continuación. Las prácticas de planificación de estudio detectadas se describen en las categorías 1, 2, 3, 4, 5, 6 y 7 . Las prácticas de monitoreo de estudio se describen en las categorías 8,9,10,11 y 12. Las prácticas de evaluación de estudio se describen en las categorías 13,14,15, 16 y 17. El guión del grupo de discusión se baso en la teoría de Zimmerman (2000, 2002). 


\section{Categoría 1: Planificación del estudio}

Los estudiantes refieren que organizan el estudio, sin especificar cómo lo realizan. También plantean que existen dificultades para organizarse debido a lo menos tres elementos de significado: la sobrecarga de labores académicas, las distracciones y las preocupaciones. Las técnicas que utilizan para estudiar son: lectura de diapositivas de las clases, confección de resúmenes, lecturas con diferentes niveles de profundidad, usando sólo el subrayado. Así también, plantean que la mejor forma de aprender es atender en las clases, sobre todo los ejemplos.

\section{Categoría 2: Organización del Estudio}

Sólo dos estudiantes que asistieron a un programa de organización del estudio en Facultad de Medicina en UCN, plantean que aprendieron a organizarse y concentrarse, así como también a realizar lecturas de diferentes profundidades.

\section{Categoría 3, 4: Técnica para leer textos/Dificultad para leer}

Los estudiantes plantean que el tener gusto por la lectura ayuda a estudiar, es decir, en algunos casos si existe mayor disposición a leer textos de gusto personal, aquello facilitaría la adquisición del hábito lector como herramienta para estudiar.

Categorías 5, 6, 7: estudio individual y grupal/cómo es el trabajo grupal/ elección estudio grupal o individual Los estudiantes plantean que al inicio es complejo estudiar en grupo, dado que se necesita que los integrantes hayan leído previamente toda la información y así estén todos en condiciones de compartir. Según expresan los estudiantes, prefieren el estudio grupal, porque es dinámico y se construye con las otras personas y que el estudio grupal es mejor a corto plazo y el individual es mejor a largo plazo.

\section{Categoría 8: Esfuerzo e impacto en los resultados}

Los estudiantes manifiestan que no esforzarse incidirá directamente en la calificación (corto plazo) y además, en no tener una buena base para su futuro profesional (largo plazo). No indican cómo evalúan el esfuerzo.

\section{Categoría 9: preparación, evaluaciones y malos resultados}

Los estudiantes plantean que si sólo se estudia para la prueba se obtendrán malas calificaciones. Por otro lado, plantean que al momento de prepararse, existen diversos factores que afectan el logro de los resultados en una prueba, tal como: los nervios, presión, distracciones, cansancio, deseo o no por estudiar. Se plantea también, que a más tiempo de estudio, no necesariamente asegura buenos resultados, sino más bien estudiar mejor.

Categoría 10, 11: confianza en capacidades personales y su incidencia en resultados/importancia de la calificación

Los estudiantes plantean que el tener confianza y seguridad afectan los resultados obtenidos, dado que si dudan en una evaluación o están preocupados por otra cosa, eso afecta las respuestas dadas. Se plantea que lo importante es aprender y no la calificación.

Categoría 12: Traspaso de técnica de estudio a otras asignaturas

Los estudiantes plantean que estudian de la misma manera para todas las asignaturas.

Categoría 13, 14: Incidencia de la disposición positiva y resultados/ resultados negativos versus próxima evaluación

Los estudiantes plantean que la confianza se ve afectada por la negatividad, y que la preocupación excesiva provoca problemas de concentración. También expresan que cuando se obtienen resultados negativos se debe estudiar más y prestar dedicación al estudio.

Categoría 15: Concepto de dedicación y resultados en los estudios

Los estudiantes plantean que se debe invertir mayor tiempo en aquello que no se maneja bien. Además, analizar en que se falló, establecer relación de los aprendizajes logrados entre una y otra prueba.

Categoría 16: Responsabilidad en el resultado del estudio

Los estudiantes plantean que la responsabilidad es compartida entre el estudiante y el docente.

Categoría 17: Confianza y resultados del estudio

Los estudiantes plantean que los docentes pueden influir en la confianza de los estudiantes, a través de tranquilizar, animar o desorientar. 


\section{DISCUSIÓN}

\section{Prácticas de planificación de estudio}

Las categorías 1, 2, 3, 4, 5, 6 y 7 respondieron a las prácticas de estudio detectadas en los estudiantes. Cabe destacar que en esta fase, se puede plantear que los estudiantes no organizan su proceso de estudio. Si bien explicitan que lo planifican, no logran especificar el cómo lo realizan. Sin embargo, informan que el planificar resulta difícil debido a la sobrecarga académica, las distracciones previas al estudio, las preocupaciones personales, los nervios, presión, cansancio y deseo de no estudiar. Con referencia a las técnicas utilizadas, los estudiantes utilizan la lectura de diapositivas, resúmenes, lecturas con diferentes niveles de profundidad, subrayado, atención en clases. Así mismo plantean que el gusto por la lectura se debe practicar, primero con lecturas de interés personal, y así transferir el hábito lector a las lecturas de las asignaturas. En relación al estudio en grupo, existe una valoración positiva, dado que es dinámico y pueden construir con sus compañeros. Sin embargo, plantean que existen condiciones previas que se deben cumplir para que sea positivo: todos deben tener el mismo nivel de conocimiento sobre la materia, dado que si no es así se dificulta el inicio del trabajo grupal. Por lo anterior, se aprecia que existe una intensión de planificación del estudio por parte de los estudiantes, sin embrago, no hay ninguna forma concreta de organización declarada por ellos. Entonces, en la intervención docente para promover el aprendizaje autorregulado será necesario considerar que ya existe una sobrecarga planteada por los estudiantes por lo cual el trabajo de entrenamiento de autorregulación del aprendizaje debe realizarse en la clase misma y no como un programa extracurricular. También será importante hacer consciente el proceso de autorregulación y sus tres etapas.

\section{Prácticas de monitoreo del estudio}

Las categorías 8,9,10,11 y 12 respondieron a las prácticas de monitoreo del estudio detectadas en los estudiantes. Con referencia al análisis de haber preparado el estudio y luego obtener malos resultados en la evaluación, los estudiantes plantean que no es adecuado prepararse sólo para la prueba, dado que se obtendrán malas calificaciones. Así también hacen referencia a que estudiar más, no asegura buenos resultados, sino que "estudiar mejor" aportaría a lograr buenos resultados. En relación a cómo impacta el esfuerzo en los resultados obtenidos, los estudiantes manifiestan que si no se esfuerzan esto afectará la calificación en el corto plazo, y que a largo plazo se verá afectada la base para el ejercicio de la profesión.

Con referencia al cambio de técnica de estudio al obtener malos resultados, se plantea que debería analizarse por qué se obtuvieron esos resultados, y así decidir qué hacer, considerando la naturaleza de la asignatura y de cómo evalúa el docente, comenzando así un cambio gradual, sin embargo no se indica cómo se debería realizar ese cambio. En relación a cómo afecta la confianza personal en los resultados del estudio, los estudiantes refieren que el dudar de sus capacidades, afecta el resultado en una evaluación.

Como se pudo apreciar en las respuestas de los estudiantes no existe un mecanismo formal y sistemático que permita realizar un monitoreo de su estudio ya que se indica que se debe cambiar de técnica de estudio, pero no se hace mención de ninguna técnica de estudio.

\section{Prácticas de evaluación del estudio}

Las categorías 13,14,15, 16 y 17 respondieron a las prácticas de evaluación del estudio declaradas por los estudiantes. Con referencia a cómo se enfrentan a los malos resultados, se plantea que se debe analizar en qué se fallo y persistir en el estudio. Se debe invertir mayor tiempo en aquello que no se aprendió bien. Relacionar los aprendizajes entre una y otra evaluación de una misma asignatura. Así como también los resultados se ven afectados por la negatividad y la preocupación, ya que afectan la capacidad de concentración. Sin embargo, no existe una forma clara que indique cómo se podría remediar un mal resultado, ya que no hay conocimiento previo consciente de técnicas que ayuden a fomentar aprendizajes activos y autorregulados. En relación a la responsabilidad en los resultados obtenidos, se observó que los estudiantes indican que las responsabilidades son compartidas con el docente. Dado que por un lado el estudiante debe tener auto-aprendizaje y auto-crítica. Por otro lado el docente debe ser un guía, quien explica la utilidad de lo aprendido, anima y tranquiliza a los estudiantes. Lo anterior es importante ya que existe conciencia y reflexión a cerca de la responsabilidad compartida en el proceso de enseñanza aprendizaje.

En términos generales al aplicar el grupo de discusión se observó que muchas de las respuestas recibidas no se relacionaban directamente con el tema consultado. Se observó saltos de un tema a otro, logrando poca profundidad en las respuestas de los estudiantes. Se observó que los aspectos emocionales relacionados con el aprendizaje, surgen constantemente en el discurso, desde la primera hasta la última categoría. Sin embargo, y no menos importante es que los estudiantes son capaces de reflexionar sobre cómo estudian, y cómo se relaciona esto con los resultados y las responsabilidades existentes en los resultados. 


\section{DOCENCIA PARA PROMOVER EL APRENDIZAJE AUTORREGULADO}

Debido al diagnóstico realizado se buscó modelar una estrategia docente que permitiese utilizar técnicas que fomenten el aprendizaje autorregulado en la asignatura de Ingeniería de Software. Por otro lado en el caso de Autoeficacia General no se detectaron mayores problemas según las respuestas entregadas por los estudiantes, lo que indicaría que los estudiantes se perciben autoeficaces y motivados por la carrera que estudian. Lo anterior les permite mantener una actitud positiva, sin embargo la carencia de estrategias de aprendizaje autorregulado es evidente. Por otro lado el trabajo de Pérez et. al. (2010) propone un programa para facilitar el aprendizaje activo y autorregulado, este programa no esta asociado a una asignatura en particular. En el caso de este trabajo se optó por comenzar, a modo de piloto en una sola asignatura, con el siguiente plan: (1) Planificación de Estudio; para este caso se utilizó el cuadro CQA, se registro la planificación de objetivos de aprendizaje y se gestionó el tiempo en los talleres de promoción del aprendizaje autorregulado.(2) Monitorear Estudio, se aplicó estrategias de aprendizaje tales como guías de estudio y mapas conceptuales propios de la especialidad de ingeniería de software (UML). (3) Luego, para Evaluar Estudio se aplicó coevaluación y autoevaluación. La Figura 2 resume los focos de la promoción del aprendizaje autorregulado en el curso de Ingeniería de Software.

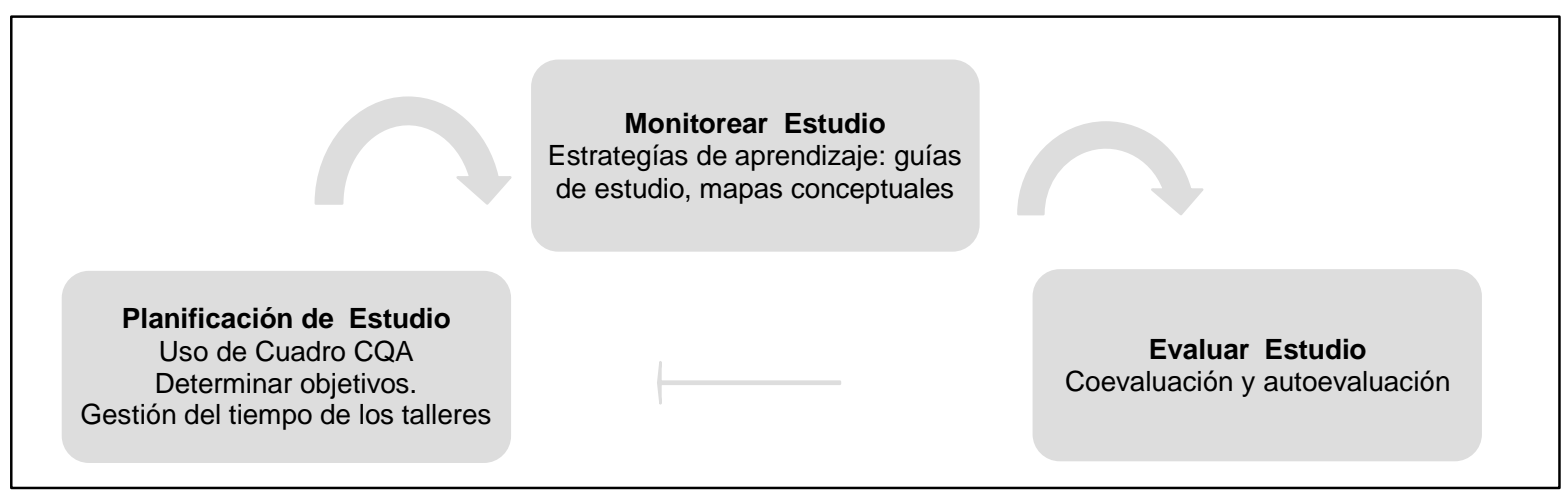

Fig. 2: Focos de la promoción del aprendizaje autorregulado en el curso de Ingeniería de Software

Por otro lado, como sustento al hecho de que a los estudiantes a los que sus profesores enseñan y modelan de forma sistemática estrategias de autorregulación de aprendizaje ejercitan más automáticamente su utilización, encontramos los trabajos de Ablard y Lipschultz (1998).

Para este estudio se establecieron los siguientes indicadores para evaluar el trabajo docente sobre el fomento del aprendizaje autorregulado (ver Figura 3).

\begin{tabular}{|c|c|c|c|}
\hline \multirow{2}{*}{ Impacto } & $\begin{array}{c}\text { Estudiantes que } \\
\text { aprueban el curso }\end{array}$ & \multirow{2}{*}{$\begin{array}{c}\text { Indicador } \\
\text { Satisfacción }\end{array}$} & $\begin{array}{c}75 \% \text { de los estudiantes } \\
\text { aprueban el curso }\end{array}$ \\
\cline { 2 - 2 } & $\begin{array}{c}\text { Estudiantes que utilicen } \\
\text { estrategias de } \\
\text { aprendizaje } \\
\text { autorregulado }\end{array}$ & $\begin{array}{c}75 \% \text { de los estudiantes } \\
\text { utilizan estrategias de } \\
\text { aprendizaje } \\
\text { autorregulado }\end{array}$ \\
\hline
\end{tabular}

Fig. 3: Indicadores para evaluar la promoción del aprendizaje autorregulado

Cuadro CQA: La técnica del cuadro CQA fue desarrollada por Ogle (1986) para activar el conocimiento previo de los estudiantes y ayudarles a determinar sus propósitos frente a los temas que se estén estudiando. Requiere que los estudiantes focalicen su atención en tres preguntas: dos antes de leer lo que se desea estudiar y una después de leer lo estudiado: ¿Qué sé sobre este tema? (C), ¿Qué quiero aprender? (Q) y ¿Qué he aprendido? (A). Es importante indicar que las dos preguntas hechas antes de leer lo que se desea estudiar activan el conocimiento previo de los estudiantes y establecen sus propósitos frente a lo estudiado, generando preguntas que ellos desean responder. De esta forma el cuadro CQA se presenta como una herramienta muy útil para planificar, organizar, llevar a cabo y evaluar el estudio.

Mapas Conceptuales propios de la Disciplina Ingeniería de Software: se utilizan los diagramas de Unified Modeling Language (UML) con la metodología RUP (que permite llevar a cabo la planificación, desarrollo,testing y gestión de un proceso de desarrollo de software). 
Guías de Estudio: La Guía de estudio es una herramienta que ayuda al estudiante a preparar un(os) contenido(s) asociados a un resultado de aprendizaje. La Figura 4 muestra la estructura de una Matriz de cuadro CQA

\begin{tabular}{|c|c|c|}
\hline C & Q & A \\
¿Qué conozco del tema ? & ¿Qué quiero aprender? & ¿Qué aprendí? \\
\hline $\begin{array}{c}\text { Objetivo: Activación de } \\
\text { conocimientos previos. }\end{array}$ & $\begin{array}{c}\text { Objetivo: Formulación de Preguntas } \\
\text { previas y formulación de propósitos } \\
\text { para el aprendizaje. }\end{array}$ & $\begin{array}{c}\text { Objetivo: Dar respuesta a las siguientes } \\
\text { preguntas ¿Fue el propósito logrado? } \\
\text { ¿Quedaron todas las preguntas respondidas? } \\
\text { ¿Qué falta aprender? }\end{array}$ \\
\hline
\end{tabular}

Fig. 4: Matriz de cuadro CQA

Para promover el aprendizaje autorregulado se diseñaron 8 talleres. En cada taller se utilizó el cuadro CQA como herramienta para planificar, organizar y reflexionar sobre el estudio. Se entrenó al estudiante para planificar, monitorear y evaluar su estudio. Los talleres se diseñaron cuando se implementó la planificación didáctica de la asignatura Ingeniería de Software. Por lo cual de las 16 semanas que tiene el semestre para dictar clases se consideró que semana por medio se realizaría un taller. Para realizar los talleres los estudiantes debían leer un capítulo del libro o un artículo o resolver un caso práctico preparado por la docente. Así durante la clase los estudiantes debían trabajar en equipo y/o en forma individual para obtener un producto. No se asignaron los talleres como tarea fuera del aula ya que no se quería sobrecargar a los estudiantes, pues ellos mismos habían indicado en el diagnóstico que se encontraban recargados. Es importante indicar que los talleres apuntan a los contenidos que en el programa de asignatura son teóricos. De esta forma se trabajan en modalidad de aprendizaje activo y no de forma tradicional por la exposición de diapositivas. Durante el desarrollo del taller de promoción del aprendizaje autorregulado se siguen los siguientes pasos:

i) Se entrega el cuadro CQA al inicio de la clase para que los estudiantes lo completen con los conocimientos previos.

ii) Luego se explica la técnica (que potencie el aprendizaje autorregulado) y se realiza un ejemplo usando la técnica de tal forma que el estudiante pueda aplicarla al contexto que estudia. El grupo donde trabaja el estudiante debe entregar un informe al final de la clase.

iii) Se entrega el cuadro CQA al final de la clase para que el estudiante lo complete con lo aprendido durante el desarrollo de la clase.

iv) En la clase siguiente se entrega la pauta con el taller corregido para que un grupo distinto realice las correcciones del taller de otro grupo. También se entrega el cuadro CQA para evaluar. Finalmente se emite un breve informe con el resultado final del taller que sirve como retroalimentación para los estudiantes y además permite agregar un compromiso de mejora para cada grupo de trabajo.

La Figura 5 muestra un ejemplo de Taller de Promoción del Aprendizaje Autorregulado. Por otro lado es importante indicar que el resto de las clases se trabaja en el desarrollo del proyecto de desarrollo de software para entregar las competencias relacionadas con los resultados de aprendizaje asociados al proyecto del curso.

Taller de Promoción del Aprendizaje Autorregulado

Objetivo: Desarrollar una guía de estudio sobre metodologías ágiles de desarrollo de software.

Previa lectura del artículo "Revisión de metodologías ágiles para el desarrollo de software", asignado la clase pasada. Trabaje durante la clase para elaborar lo que se pide a continuación.

\begin{tabular}{|l|l|l|}
\hline Etapa de la clase & Tiempo & Actividad desarrollada \\
\hline Inicio de la clase & 10 minutos & $\begin{array}{l}\text { Complete el cuadro CQA - Los estudiantes sólo completan las letras C y Q del cuadro } \\
\text { CQA. }\end{array}$ \\
\hline Durante la clase & 70 minutos & $\begin{array}{l}\text { Actividad Central a desarrollar durante la clase - Desarrolle una guía de estudio con } \\
\text { las principales características de las metodologías ágiles. Explique cómo podría } \\
\text { adecuar una de las metodologías ágiles a su proyecto de desarrollo de software. }\end{array}$ \\
\hline Fin de la clase & 10 minutos & Complete el cuadro CQA - Los estudiantes completan la letra A del cuadro CQA. \\
\hline
\end{tabular}

Fig. 5: Ejemplo de Taller de Promoción del Aprendizaje Autorregulado 
Como se indicó anteriormente la clase se estructuró y planificó de tal forma que el estudiante debe realizar un proceso de enseñanza aprendizaje bajo las tres etapas del aprendizaje autorregulado, es decir, planificación, monitoreo y evaluación. Por otro lado el nivel de satisfacción de los estudiantes fue elevado al aprobar la asignatura y tomar conciencia del proceso de aprendizaje autorregulado y las consecuencias positivas en su vida académica y laboral.

\section{CONCLUSIONES}

Según el análisis, los resultados y la propuesta de promoción de aprendizaje autorregulado presentados en este trabajo se concluye que:

En general, como resultado se obtuvo que los estudiantes no organizan, ni planifican su proceso de estudio. Tampoco existe una forma explícita ni clara de monitorear su proceso de aprendizaje. Debido a estos resultados se hizo necesario modificar la planificación didáctica del curso ingeniería de software para incluir 8 talleres de promoción del aprendizaje autorregulado. Los talleres de promoción del aprendizaje autorregulado permitieron a los estudiantes instruirse en técnicas de estudio para la autonomía y también permitieron organizar la clase en un formato de aprendizaje activo.

Por otro lado el diseño de la intervención pedagógica permitió promover el aprendizaje autorregulado y es fácilmente replicable a otras asignaturas y/o carreras. Lo anterior se desarrolló diseñando talleres de promoción del aprendizaje autorregulado usando cuadro CQA, guías de estudio y mapas conceptuales propios de la disciplina ingeniería de software. El resultado de la intervención pedagógica proporciono a los estudiantes herramientas para organizar, monitorear y evaluar el proceso de aprendizaje autorregulado de forma consciente. También es importante indicar que se cumplieron los indicadores establecidos al comienzo de la innovación pedagógica, es decir, más del $75 \%$ de los estudiantes aprueban el curso y mas del $75 \%$ de los estudiantes utilizan estrategias de aprendizaje para aprender autónomamente.

\section{AGRADECIMIENTOS}

Se agradece a Valeria Burgos y Katherine Campusano de CIMET Coquimbo, María Morales, Secretaria de Investigación UCN Coquimbo y Marcos Chait académico Escuela de Ingeniería por el apoyo en esta investigación. Este trabajo obtuvo el premio a la Innovación Docente año 2015 en UCN.

\section{REFERENCIAS}

Ablard K., Lipschultz, R. Self-regulation in high-achieving students: relations to advanced reasoning, achievement goals, and gender. Journal of Educational Psychology, 90(1), 94-101, (1998)

Baessler, J., Schwarzer, R. Evaluación de la autoeficacia: Adaptación española de la Escala de Autoeficacia General. Ansiedad y Estrés, 2(1), 1-8, (1996)

Biggs, J. The reflective institution: assuring and enhancing the quality of teaching and learning. Higher Education, 42, 221-237, (2001)

Bouffard, T., Bouchard, M., Goulet, G., Denoncourt, I., Couture, N. Influence of achievement goals and selfefficacy on students' selfregulation and performance. International Journal of Psycologhy, 40(6), 373-384, (2005)

Cid, H., Orellana, Y., Barriga, O. Validación de la escala de autoeficacia general en Chile, Revista Médica de Chile, 138(5), 551-557 (2010)

Cochran-Smith, M. Teaching quality matters. Journal of Teacher Education, 54(2), 95-98, (2003)

Díaz, A., Pérez, M.V., Valenzuela, M., Muñoz, P., Rivas, S., Salas, C. Procesos de autorregulación del aprendizaje en estudiantes universitarios de primer año. Internacional Journal of Developmental an Educational Psychology, 4(1), 789-800, (2010)

Daura, F. Las estrategias docentes al servicio del desarrollo del aprendizaje autorregulado. Estudios pedagógicos, 37(2), 77-88, (2011)

Daura, F. Aprendizaje autorregulado y rendimiento académico en estudiantes del ciclo clínico de la carrera de Medicina. Revista Electrónica de Investigación Educativa, 17(3), 13-27, (2015) 
Entwistle, N., Tait, H. Promoting effective study skills, Effective learning and teaching in higher education. Universities' and Colleges' Staff Development Agency, Sheffield, UK (1992)

Monereo, C., Badia, A., Bilbao G., Cerrato, Ma., Weise, C. Ser docente estratégico: cuando cambiar la estrategia no basta. Cultura y Educación, 21 (3), 1-20, (2009)

Namakforoosh, J.M. Metodología de la investigación, 1-528, Editorial Limusa, México, (2011)

Ogle, D.M. K-W-L: A teaching model that develops active reading of expository text. Reading Teacher, 39, 564-570. (1986)

Pérez, M.V., Díaz, A., González, J., Nuñez, J. Docencia para facilitar el aprendizaje activo y autorregulado. Revista Dialogo Educacional Curitiba, 10(3), 409-424, (2010)

Pintrich, P., Smith, D., García, T., Keachie, W. A manual for the use of the Motivated Strategies for Learning Questionnaire (MSLQ). Michigan: National Center for Research to Improve Postsecondry Teaching and Learning. University of Michigan. (1991)

Pintrich, P., De Groot, E.V. Motivational and Self-Regulated Learning Components of Classroom Academic Performance. Journal of Educational Psychology, 82(1), 33-40, (1990)

Pool-Cibrián, W., Martínez-Guerrero, J. Autoeficacia y uso de estrategias para el aprendizaje autorregulado en estudiantes universitarios. Revista Electrónica de Investigación Educativa, 15(3), 21-37, (2013)

Rosario, P., Nuñez, J., Gonzalez-Pienda, J. Stories that show how to study and how learn: an experience in Portuguese school system. Electronic Journal of Research in Educational Psychologist, 2(1), 131- 144, (2004).

Unified Modeling Language, UML, http://www.uml.org/index.htm, acceso 2 de febrero 2016 (2016).

Zeidner, M., Boekaerts, M., Pintrich, P.R., Self-regulation: Directions and challenges for future research. Handbook of Self-Regulation, San Diego: Academic Press, 749-768, (2000).

Zimmerman, B. J., Kitsantas, A. Reliability and validity of Self-efficacy for Learning Form (SELF) scores of college students. Zeitschrift für Psychologie-Journal of Psychology, 215(3), 157-163, (2007).

Zimmerman, B. J. Self-regulated learning and academic achievement: An overview. Educational Psychologist, 25, 3-17 (1990)

Zimmerman, B. J. Attaining self-regulation: a social cognitive perspective. En M. Boekaerts, P. Pintrich y M. Zeidner (Eds.). Self-regulation: theory, research and application, 13-39, FL: Academic Press, Orlando, (2000)

Zimmerman, B. J. Theories of self-regulated learning and academic achievement: An overview and analysis. En B. J. Zimmerman y D. H. Schunk (Eds), Self-regulated Learning and Academic Achievement: Theoretical Perspectives, 1-37, London: Lawrence Erlbaum, (2001)

Zimmerman, B. J. Becoming a self-regulated learner: an overview. Theory into Practice, 41(2), 64-70, (2002)

Zimmerman, B.J. A social cognitive view of selfregulated academic learning. Journal of Educational Psychology, 81(3), 329-339, (1989)

Zambrano, C., Varas, M., Urrutia, A. Enfoque MDA para el diseño de un data warehouse difuso. Ingeniare Revista Chilena de Ingeniería, 20(1), 99-113, (2012)

Zambrano, C., Rojas, D., Carvajal, K., Acuña, G. Análisis de rendimiento académico estudiantil usando data warehouse y redes neuronales. Ingeniare Revista Chilena de Ingeniería, 19(3), 369-381, (2011) 